Apoorva, Priyanka*, Himanshu Wadhwa and Gurjit Kaur

\title{
Performance Analysis of Hybrid Optical Amplifiers for 32 Channel WDM System at 10 Gbps Bit Rate for WAN Applications
}

https://doi.org/10.1515/joc-2017-0139

Received August 16, 2017; accepted October 9, 2017

\begin{abstract}
In this paper, we have simulated an optical 32 channel WDM system with 10 Gbps data rate for various hybrid amplifiers at varying lengths. Stand-alone EDFA gives better performance than other individual amplifiers but when hybrid with Raman amplifier, the performance of the WDM system is enhanced. A lot of researchers are working on the hybrid amplifiers like EDFA and Raman. In this research paper we have also included latest amplifier such as Ytterbium and Praseodymium amplifiers and the performance of the various hybrid combinations is compared. So, different hybrid amplifiers such as RamanEDFA, EDFA-Yb, Raman-Yb, EDFA-Pr and Raman-Pr have been analyzed for performance parameters like quality factor, bit error rate, eye height and channel power. The analysis shows that EDFA-Pr has maximum Q-factor of 15.4883 at $80 \mathrm{~km}$. EDFA-Pr has a maximum eye opening of 0.00534 at $80 \mathrm{~km}$ of length but at $120 \mathrm{~km}$ transmission distance EDFA-Yb shows maximum eye opening of 0.00154. Below $100 \mathrm{~km}$ of transmission distance, EDFA-Pr shows minimum BER value ranging from $10^{-54}$ to $10^{-20}$ but as the length is increased above $100 \mathrm{~km}$ EDFA-Yb shows better performance with values ranging from $10^{-16}$ to $10^{-09}$.
\end{abstract}

Keywords: WDM system, Q-factor, BER, Eye height, Hybrid amplifiers (EDFA, Raman, Ytterbium, Praseodymium)

\section{Introduction}

In the recent era, which is flooded with new advents in technology, the need of extremely large bandwidth is increasing at a very high rate as new communication services have been introduced like video conferencing and

*Corresponding author: Priyanka, School of ICT, Gautam Buddha University, Greater Noida 201310, Uttar Pradesh, India, E-mail: priyanka.vishwakarma13@gmail.com Apoorva: E-mail: apoorva0893@gmail.com, Himanshu Wadhwa: E-mail: himanshuwdhw@gmail.com, Gurjit Kaur: E-mail: gurjeet_kaur@rediffmail.com, School of ICT, Gautam Buddha University, Greater Noida 201310, Uttar Pradesh, India
HDTV. In telecommunication research, data security and reliable transmission media with the large information carrying capacity have been the driving force. In 1978, first fiber-optic communication system was developed which reportedly supported transmission of signals at $100 \mathrm{Mbps}$ using multimode fiber operating near $850 \mathrm{~nm}$. This was followed by the introduction of the single-mode fiber which has propelled the system capacity from Mbps to Gbps with repeater spacing more than $50 \mathrm{~km}$ with increase in the wavelength of system operation to $1550 \mathrm{~nm}$ which is termed as the $\mathrm{C}$ Band, i. e. the conventional band being the spectral window from $1525 \mathrm{~nm}$ to $1565 \mathrm{~nm}$. The propagation distance has increased owing to lower attenuation but large fiber dispersion at $1550 \mathrm{~nm}$ has identified fiber dispersion as the next obstacle which has to be dealt with. Several systems were developed with reduced dispersion which could operate in the region of $10 \mathrm{Gbps}$ with repeater spacing as large as $100 \mathrm{~km}$ [1]. Now-a-day, optical communication has been enhanced to $100 \mathrm{Gbps}$ and further research is now focused to develop an optical fiber with data rates up to terabytes per second. Advances in technology have enabled more data to be traversed through a single optical fiber over long distances. The transmission capacity in optical communication networks has been significantly improved using wavelength division multiplexing (WDM) [2]. Since, optical signal is more efficient than electrical signal therefore required features for future optical networks is the ability to process information entirely in the optical domain for the following purposes: multiplexing, de-multiplexing, amplification, filtering, switching, etc. WDM has become the preferred transmission technology for transporting the optical signal over long distances [3]. In WDM system, the following amplifiers can be used: RAMAN, EDFA, SOA, Ytterbium-doped amplifier and Praseodymium-doped amplifier (new in the field of research). The development of efficient and powerful optical amplifiers has played a huge role in promoting optical communications eliminating the need of costly conversions from optical to electrical signal and vice-versa. Due to the recent introduction of wideband optical amplifiers, DWDM systems having transmission rates in Tbps have become a reality [4]. Raman amplifiers improve the noise figure and reduce the nonlinearity of fiber systems, this improves the 
overall system performance thus allows us longer amplifier spans, higher bit rates, less channel spacing (tailor made for WDM and DWDM systems) [5]. EDFA is an optical amplifier that uses a doped optical fiber to amplify an optical signal. The signal which is to be amplified is multiplexed into the doped fiber, and the signal is amplified through interaction with the doping ions. EDFA is the best known and most frequently used optical amplifier because it provides low losses in silicabased fiber [6]. To meet the demand for an increasing number of channels, high power is required at the output of each amplifier. Therefore, to fulfill the requirement of high power, cascade or multistage amplifiers can be used. To compensate the merits and demerits of different amplifiers, hybrid configurations of these amplifiers can be formed. Hybrid amplifiers are better than individual amplifiers in various aspects like wide gain bandwidth and more flat gain profile. Hybrid amplifiers even provide high power gain [7]. Ytterbium-doped fiber amplifier offers broad-gain bandwidth and a high efficiency. They have long lifetimes, can generate short pulses and can also easily be incorporated into the material used to make the laser. Praseodymium $\mathrm{Pr}^{3+}$-doped fiber amplifiers (PDFAs) are expected to play an important role in upgrading $1300 \mathrm{~nm}$ optical systems that are used in almost all terrestrial optical telecommunication networks. A lot of researches are being done in the field of optical communication with the adoption of different types of systems to meet the growing demands like in [8] where S. M. Monirul Islam et al., have implemented OCDMA (Optical Code Division Multiple Access) over WDM system along with eradication of MAI (multi-access interference) by using MDW (modified double weight) codes as signature addresses. Thus, they have been able to accommodate more number of concurrent active users. Malik Efshana Bashir et al. [9] evaluated the transmission reach performance of 8 channel WDM system in the scenario of single and multiple line rate optical networks and analyzed these using various modulation formats for best quality of transmission. Navneet Dayal et al. [10] had came up with a long range cost-effective WDM FSO (free space optics) system using hybrid configuration of optical amplifiers by enhancing the signal power to obtain a better signal at the receiver in bad weather conditions. Rajesh Yadav et al. [11] investigated the family of prime codes 1D, 2D and 3D codes for optical CDMA communication system in which they have compared the proposed 3D code with 1D and 2D codes for orthogonality and cardinality of 3D codes. The significance of dimension was explained in terms of BER v/s number of users for 1D and 2D codes.
The article is organized as follows. Section 1 gives the introduction of different hybrid amplifiers and various researches done in past by different researchers. Section 2 describes the system designed by us. Section 3 describes the results and analysis for 32 channel WDM system of different hybrid amplifiers using parameters like eye height, BER and quality factor. At the end, Section 4 presents the conclusion of the research paper.

\section{Simulation setup}

In this article, we have investigated the performance of different hybrid optical amplifiers in a WDM system. The WDM system consists of a 32 channel transmitter in which an individual data rate of each user is $10 \mathrm{Gbps}$. The transmitter consists of pseudo-random bit sequence (PRBS) generator, non-return to zero (NRZ) pulse generator, Mach-Zehnder modulator and a laser source of 193.1 $\mathrm{THz}$ with $100 \mathrm{GHz}$ channel spacing as depicted in the block diagram (Figure 1).

The PRBS generator generates a random binary sequence of $10 \mathrm{Gbps}$ which is passed through NRZ pulse generator. The NRZ electrical pulse is then sent to the Mach-Zehnder modulator. The continuous wave laser of wavelength $1550 \mathrm{~nm}$ with spacing of $0.8 \mathrm{~nm}$ in 32 channels is fed into the Mach-Zehnder modulator. The signal is then fed into the $(32 \times 1)$ multiplexer and the multiplexed signal is traversed through the optical fiber for different lengths $(80-120 \mathrm{~km})$ which is first sent to dispersion compensated fiber. Earlier, when a single mode optical fiber was used, the received signal was highly dispersed. So, we used a dispersion compensated fiber. The core profile of a dispersion compensated fiber can be controlled to produce just the amount of dispersion required. The compensating fiber typically has a dispersion of around $-85 \mathrm{ps} / \mathrm{nm} / \mathrm{km}$ in the $1550 \mathrm{~nm}$ wavelength band. The dispersion acts in an opposite direction to that of the standard fiber and thus the distortion is reduced.

Optical amplifiers (OAs) boost the amplitude or add gain to optical signals passing through the fiber. The signal traverses through the hybrid amplifier and then it is demultiplexed at the receiver end with the help of a (1X2) demultiplexer. At the receiver end, the signal is sent through a PIN photo-detector with constant responsivity where the optical signal is converted into the electrical signal again and then passed through a low-pass Bessel filter with cutoff frequency $193 \mathrm{THz}$. Afterward, the signal is sent to the 3R-generator which performs the following functions, i.e. reshaping, retiming and retransmitting. The signal after 


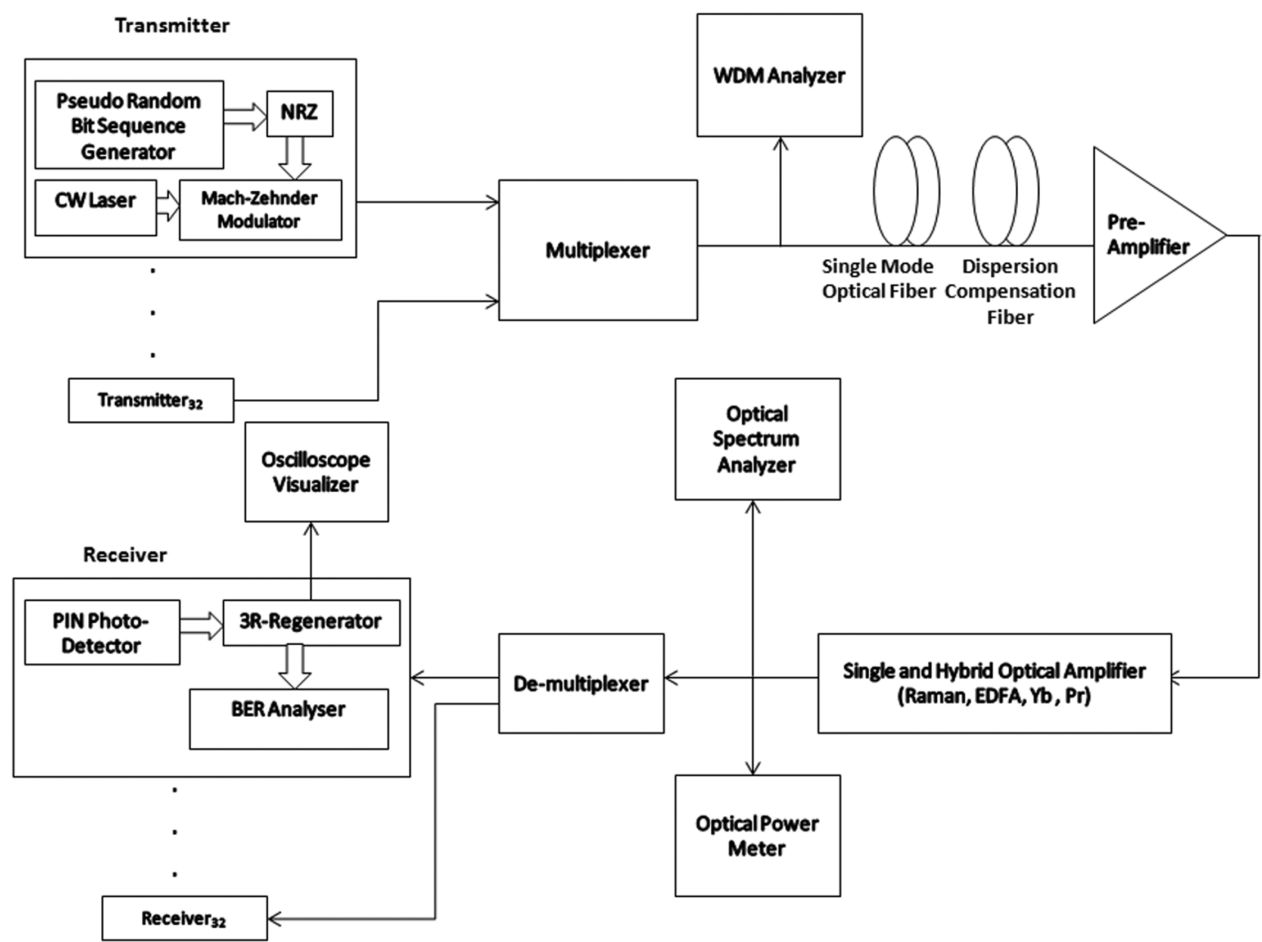

Figure 1: Block diagram of 32 channel WDM system using different hybrid optical amplifiers. performing the above functions is seen on an oscilloscope visualizer.

The performance parameters such as Q-factor, BER, and eye height are then observed at the BER analyzer which is connected to the 3R-generator.

Figure 2 shows the schematic diagram of WDM systems by using hybrid amplifiers. As the system is simulated for 32 channels, Figure 3 (a) depicts the wavelength spectrum of 32 channels. The transmitted power of all the users has been set to $0 \mathrm{dBm}$ which can be seen in Figure 3 (a). The transmitted signal from PRBS generator of $10 \mathrm{Gbps}$ data rate can be seen in Figure 3 (b). The design parameters of the above schematic are described in Table 1. Table 2 represents the hybrid optical amplifier parameters.

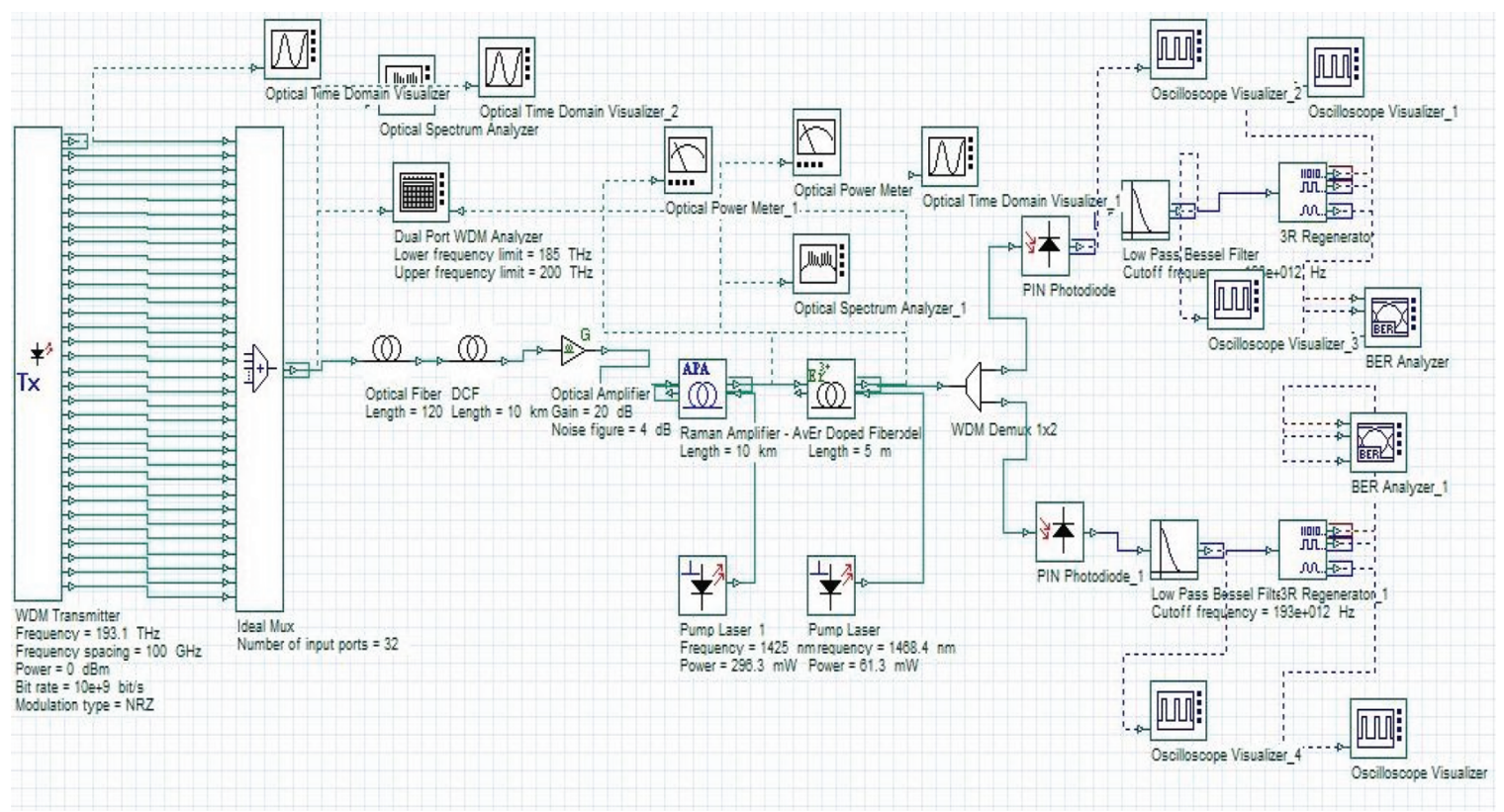

Figure 2: Schematic diagram of WDM system. 


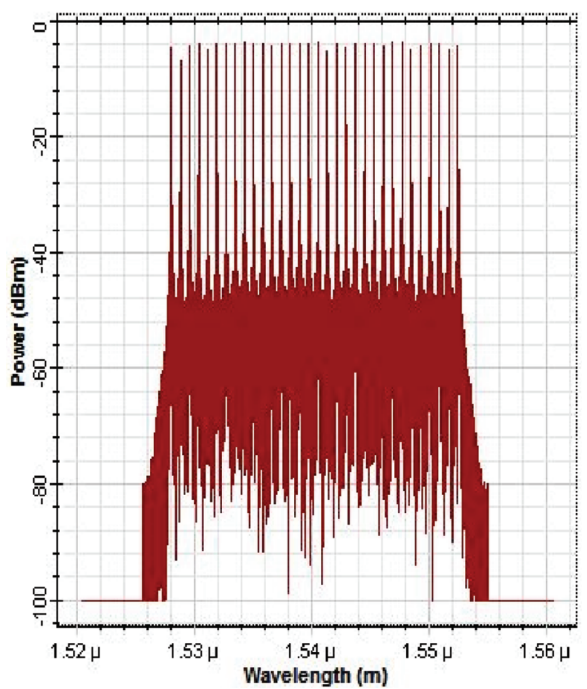

(a)

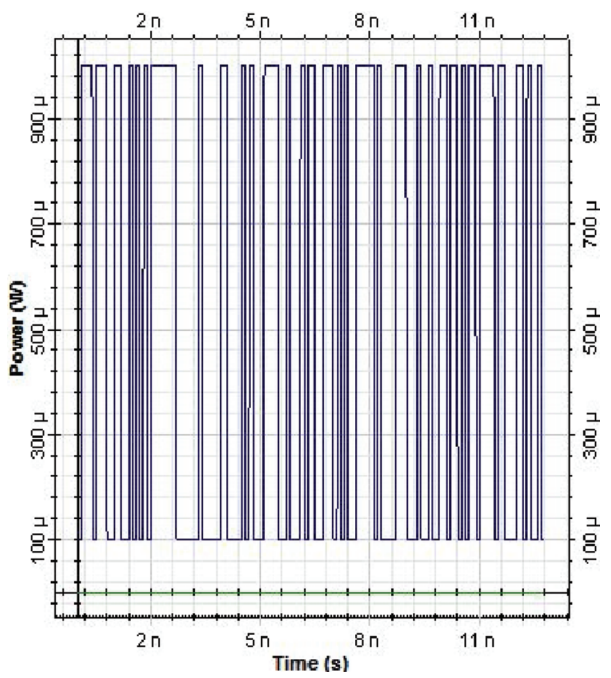

(b)
Figure 3: (a) Spectrum of 32 multiplexed optical signals; (b) transmitted signal.
Table 1: Design parameters of transmitter and receiver.

\begin{tabular}{ll}
\hline Parameters & Values \\
\hline Tx frequency & $193.1 \mathrm{THz}$ \\
Tx frequency spacing & $100 \mathrm{GHz}$ \\
Tx power & $0 \mathrm{dBm}$ \\
Tx bit rate & $10 \mathrm{e}^{+009} \mathrm{bits} / \mathrm{sec}$ \\
Sample rate & $640 \mathrm{e}^{+009} \mathrm{~Hz}$ \\
Symbol rate & $2.5 \mathrm{e}^{+009} \mathrm{symbols} / \mathrm{sec}$ \\
Rx type & PIN Photodiode \\
Rx Filter cut off frequency & $193 \mathrm{e}^{+012} \mathrm{THz}$ \\
\hline
\end{tabular}

\section{Results and analysis}

As we know, hybrid amplifiers as compared with single amplifiers help us to obtain better Q-factor, less BER and more eye height. Eye height is defined as the ratio of the height of the eye opening within three standard deviations to the amplitude. We are not only taking Raman-EDFA but we are also taking into account hybridization with $\mathrm{Yb}$ and $\mathrm{Pr}$ which have not been researched deeply yet.

\subsection{Eye height analysis of 32-channels $(120 \mathrm{~km})$}

Figure 4(a) shows eye diagram of single channel EDFA-Pr Hybrid amplifier, whereas the rest of figures i. e., 4(b) to 4(f) represent eye diagrams hybrid optical amplifiers for 32 channel WDM system. It is clearly visible that Figure 4 (a) has a wide eye opening of $0.00871 \mu \mathrm{m}$ at $120 \mathrm{~km}$ for one user; therefore, the quality of the signal is good. On the contrary, 32 channel systems show comparatively less eye height of $0.0014 \mu \mathrm{m}$ at $120 \mathrm{~km}$ which implies that the distortion in the system is increased. Among all the different combinations of hybrid amplifiers, combinations with EDFA performed better as compared to combination with Raman amplifier. Further, EDFA-Pr shows best results at $120 \mathrm{~km}$ transmission distance, whereas Raman$\mathrm{Yb}$ and Raman-Pr show highly degraded performance.

Table 2: Design parameters of hybrid optical amplifiers.

\begin{tabular}{lllll}
\hline Parameters & Raman & EDFA & Yb & Pr \\
\hline Length & $80-120 \mathrm{~km}$ & $80-120 \mathrm{~km}$ & $80-120 \mathrm{~km}$ & $80-120 \mathrm{~km}$ \\
Pump power & $296.3 \mathrm{~mW}$ & $61.3 \mathrm{~mW}$ & $61.3 \mathrm{~mW}$ & $61.3 \mathrm{~mW}$ \\
Pump wavelength & $1425 \mathrm{~nm}$ & $1468.4 \mathrm{~nm}$ & $1468.4 \mu \mathrm{m}$ & $1468.4 \mathrm{~nm}$ \\
Core radius & - & $2.2 \mu \mathrm{m}$ & $3.4 \mu \mathrm{m}$ & $1 \mu \mathrm{m}$ \\
Numerical aperture & - & 0.24 & 0.2 & 0.364 \\
\hline
\end{tabular}




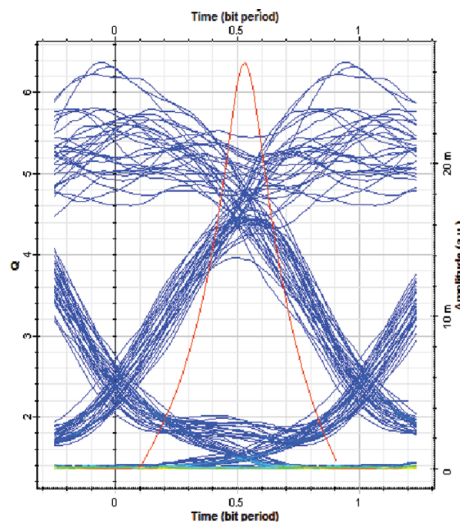

(a)

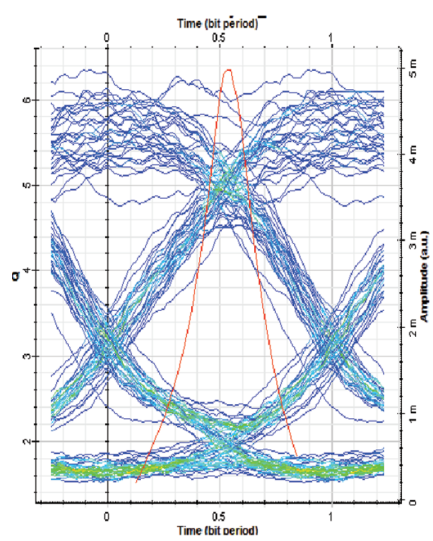

(d)

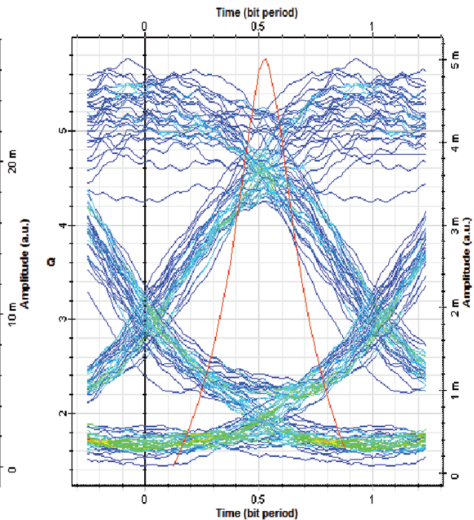

(b)

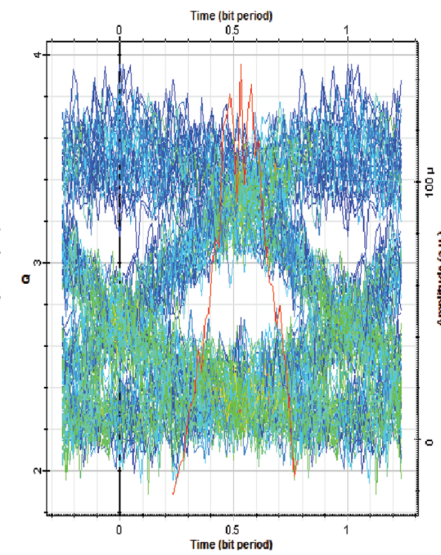

(e)

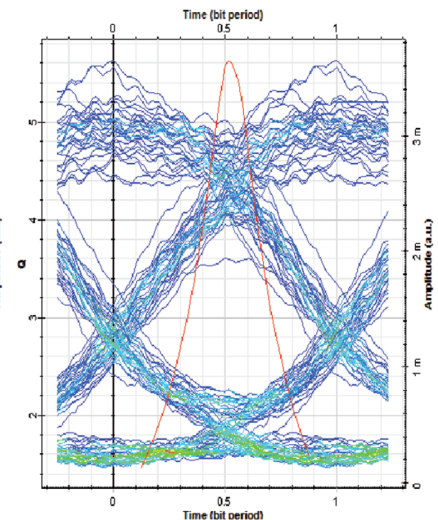

(c)

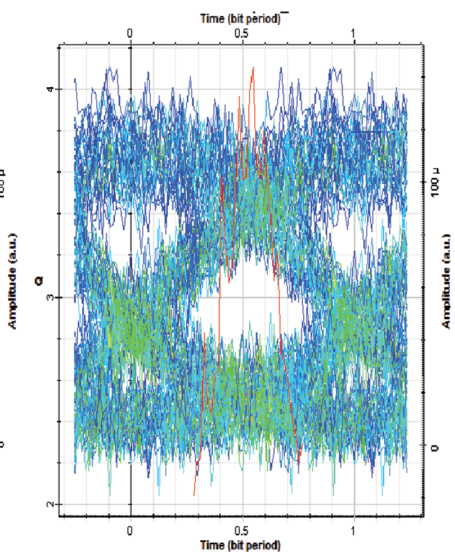

(f)

Figure 4: (a) EDFA-Pr (1-channel); (b) EDFA-Pr; (c) Raman-EDFA; (d) EDFA-Yb; (e) Raman-Yb; (f) Raman-Pr.

\subsection{Analysis of signal received $(120 \mathrm{~km})$}

Figure 5 (a)-(e) shows received signal for a transmitted signal of $1000 \mathrm{uW}$ power. Here again it can be seen that the combination with Raman shows degraded performance. A lot of noise or interference has been added in Figure 5 (a) and (e) as compared to Figure 5 (a), (b), (c). Among EDFA-Pr, EDFA-Raman, and EDFA-Yb, the EDFAPr shows better amplitude, i. e., around 5 a.u as compared to others. Further analysis shows that a significant increase in output power to $4.8 \mathrm{~mW}$ in EDFA-Pr, $4.6 \mathrm{~mW}$ in EDFA-Yb and $3 \mathrm{~mW}$ in Raman-EDFA has been observed whereas the use of Raman-Yb and Raman-Pr has been shown to degrade the performance of the system.

As we can see from Table 3 that the increase in output power is maximum for Raman-EDFA which is 5.889 . A similar but less amount of increase is observed in EDFA-YB and EDFA-Pr hybrid optical amplifiers. But Raman-Yb and Raman-Pr show a degraded performance in terms of increase in output power amounting to only 0.8 approximately.

\subsection{Analysis of length of the optical fiber vs Q-factor}

Figure 6 shows the variation of quality factor with respect to the length of fiber. It is clearly visible that EDFA-Pr has maximum Q-factor of 15.4883 at $80 \mathrm{~km}$. Raman-Yb has the least value of Q-factor, i.e., 13.072. As Figure 6 depicts that as the length of the fiber is increased from $80 \mathrm{~km}$ to $120 \mathrm{~km}$ the quality factor degrades for 15.8 to 5.6 for EDFA-Pr combination. Raman-EDFA and EDFA-Yb have approximately same values from $100 \mathrm{~km}$ to $120 \mathrm{~km}$ of length with a value of 5.9 at $120 \mathrm{~km}$.

\subsection{Analysis of length of the optical fiber vs eye height}

Figure 7 shows the variation of eye height with respect to the length of the fiber. EDFA-Pr has a maximum eye opening of 0.00534 at $80 \mathrm{~km}$ of length and Raman-EDFA 


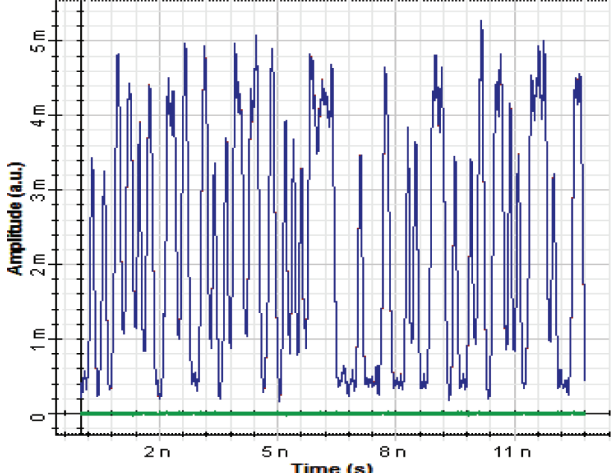

(a)

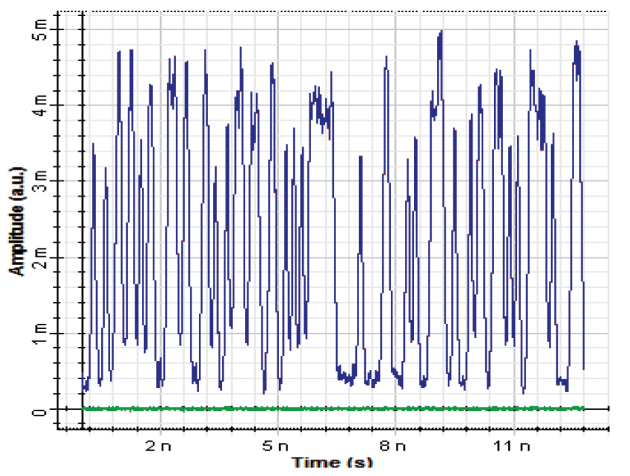

(c)

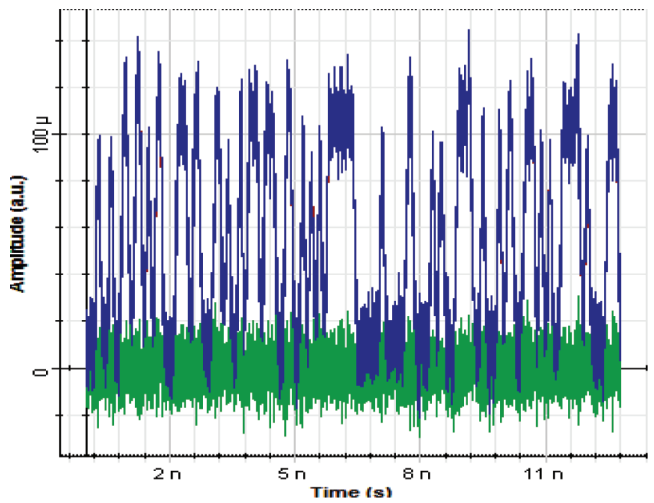

(e)

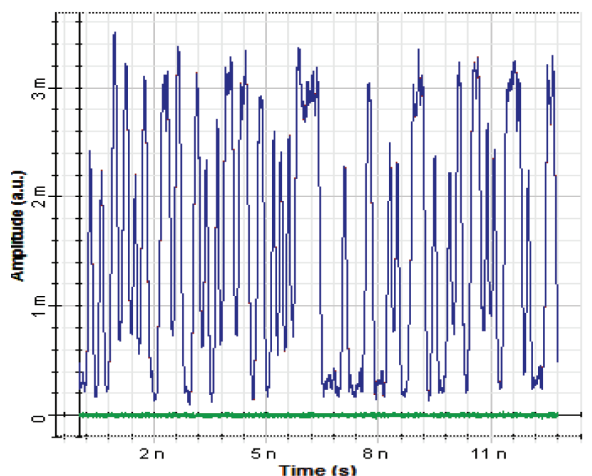

(b)

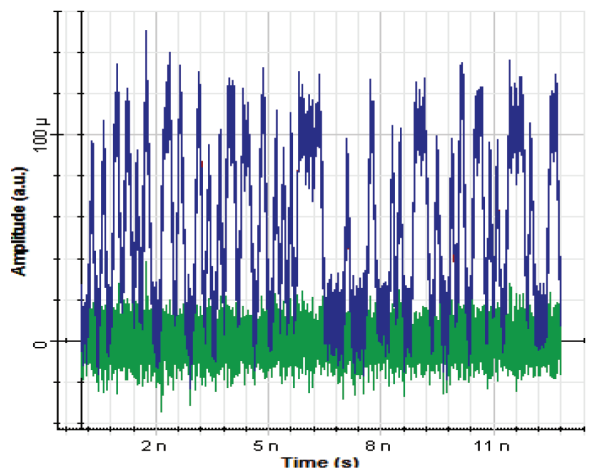

(d)

Figure 5: (a) EDFA-Pr; (b) Raman-EDFA; (c) EDFA-Yb; (d) Raman-Yb; (e) Raman-Pr.

Table 3: Analysis of power received at the end of channel.

\begin{tabular}{lrrr}
\hline Name & $\begin{array}{r}\text { Input power } \\
(\mathbf{d B m})\end{array}$ & $\begin{array}{r}\text { Output power } \\
(\mathbf{d B m})\end{array}$ & $\begin{array}{r}\text { Difference in } \\
\text { values }(\mathbf{d B m})\end{array}$ \\
\hline Raman-EDFA & 12.406 & 18.295 & 5.889 \\
EDFA-Yb & 12.406 & 17.828 & 5.422 \\
Raman-Yb & 12.406 & 13.206 & 0.8 \\
EDFA-Pr & 12.406 & 17.826 & 5.42 \\
Raman-Pr & 12.406 & 13.208 & 0.799 \\
\hline
\end{tabular}

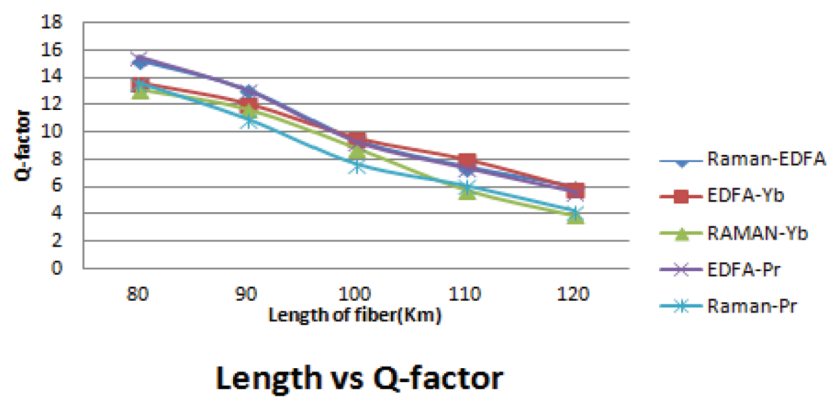

Figure 6: Length vs Q-factor. 


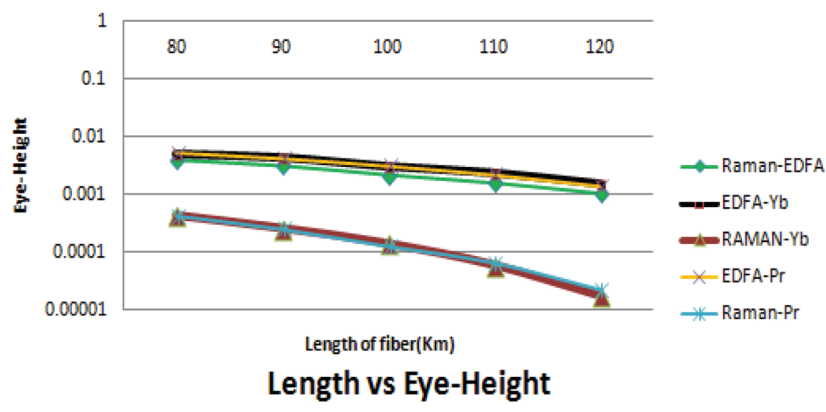

Figure 7: Length vs eye height.

has least with a value of 0.004029. As length is increased further to $120 \mathrm{~km}$ EDFA-Yb shows maximum eye opening of 0.00154. So, EDFA-Yb gives better results among all combinations.

\subsection{Analysis of length of the optical fiber vs BER}

Figure 8 shows the variation of minimum BER with the length of the fiber. The acceptable BER for optical fiber communication systems is $10^{-9}$. BER increases as the length of fiber increases. Below $100 \mathrm{~km}$ of transmission distance EDFA-Pr shows minimum BER ranging from $10^{-54}$ to $10^{-20}$ but as the length is increased above $100 \mathrm{~km}$ and EDFA-Yb shows better performance with values ranging from $10^{-16}$ to $10^{-09}$. Raman- $\mathrm{Yb}$ and Raman-Pr have highest BER, i.e., $10^{-05}$ which means that system performance degrades.

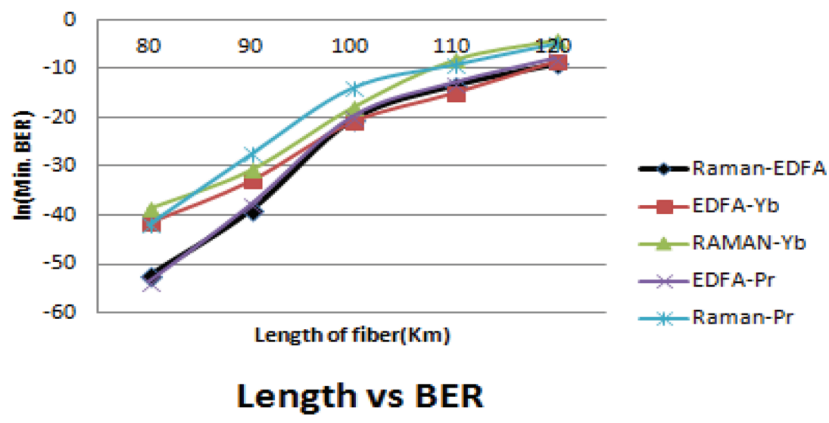

Figure 8: Length vs BER.

\section{Conclusion}

We have performed simulation of various hybrid amplifiers at varying lengths from $80 \mathrm{~km}$ to $120 \mathrm{~km}$ for WAN applications. We have thus observed that EDFA-Pr has maximum Q-factor of 15.4883 at $80 \mathrm{~km}$. Raman-EDFA and EDFA-Yb have same values from $100 \mathrm{~km}$ to $120 \mathrm{~km}$ of length with a value of 5.9 at $120 \mathrm{~km}$. In case of eye height with respect to the length of the fiber, EDFA-Pr has a maximum eye opening of 0.00534 at $80 \mathrm{~km}$ of length and Raman-EDFA has least with a value of 0.004029. At $120 \mathrm{~km}$ transmission distance EDFA-Yb shows maximum eye opening of 0.00154. In case of minimum BER with respect to the length of the fiber, BER values increase as the length of fiber increases. Below $100 \mathrm{~km}$ of transmission distance EDFA-Pr shows minimum BER value ranging from $10^{-54}$ to $10^{-20}$ but as the length is increased above $100 \mathrm{~km}$ and EDFA-Yb shows better performance with values ranging from $10^{-16}$ to $10^{-09}$. Raman- $\mathrm{Yb}$ and Raman-Pr have highest BER which increases further with length to a value of $10^{-05}$ which means that system performance degrades. So, finally our analysis concludes that at $80 \mathrm{~km}$ EDFA-Pr hybrid amplifier gives the best performance, whereas at $120 \mathrm{~km}$ EDFA-Yb hybrid amplifier is better than the rest of the hybrid configurations studied.

\section{References}

1. Arumugam M. Optical fiber communication-an overview. Pramana J Phys. 2001;57(5\&6):849-69.

2. Noshada M, Rostami A. FWM minimization in WDM optical communication systems using the asymmetrical dispersion managed fibers. Int J Light Opt. 2012;123(9):758-60.

3. Dewra S, Kaler RS. Performance evaluation of an optical network based on optical cross add drop multiplexer. J Opt Technol Aug. 2013;80(8):502-05.

4. Sun. Y. Optical amplifiers for next generation WDM optical communication networks. Microwave and Optoelectronic Conference, 1999.

5. Islam MN. Raman amplifiers for telecommunications-2 subsystems and systems. New York: Springer-Verlag, 2004:430-31.

6. Sindh UJ, Patel RB, Mehta KA, Mishra V. Performance analysis of 32-channel WDM system using erbium doped fiber amplifier. Int J Electrical Electron Eng Telecommun. 2013 April; 2(2):13-18. ISSN: 2319-2518.

7. Arora Mr G, Dewra S. A review on optical amplifier and its hybrid configurations. Int J Electr Commun Eng Res. 2013 November;1(6):1-6.

8. Islam M, Ahmed N, Ali S, Nordin JM, Sahayadhas A. Detection performance of OCDMA over WDM sytem for all optical access network. IEEE. 6-8 April 2016.

9. Bashir ME, Singh K, Devra S, Kaur G. Assessment of transmission reach of advanced modulation formats for an 8-channel wavelength division multiplexing (WDM) system in the scenario of single and mixed line rate Optical networks. Res J Eng Technol. 2017;8(3).

10. Dayal N, Singh $P$, Kaur P. Long range cost-effective WDM-FSO system using hybrid optical amplifiers. Wireless Professional Commun. 2017;1-13.

11. Yadav Dr R, Kaur G. Design and performance analysis of 1D, 2D and $3 D$ prime sequence codes family for optical CDMA network. J Opt. 2016 December; 45(4):343-56. Springer. 